\title{
Étude expérimentale des propriétés mécaniques et morphologiques dans un tube de gaz en HDPE-80
}

\author{
Nadjette Kiass ${ }^{1}$, Rabia Khelif ${ }^{2}$, Boubaker Bounamous ${ }^{2}$, Abdelaziz Amirat ${ }^{2}$ \\ ET KaMEL CHAOUI ${ }^{2, a}$ \\ 1 Laboratoire de Recherche Mécanique des Matériaux et Maintenance Industrielle (LR3MI), Département de Tronc Commun, \\ Centre universitaire (CUSA), Souk Ahras 41000, Algérie \\ 2 Laboratoire de Recherche Mécanique des Matériaux et Maintenance Industrielle (LR3MI), Département de Mécanique, \\ Université Badji Mokhtar, BP 12, Annaba 23000, Algérie
}

Reçu le 28 juin 2004, accepté le 23 août 2006

\begin{abstract}
Résumé - L'objectif de cette étude expérimentale est d'établir la distribution des propriétés mécaniques et morphologiques à travers la paroi d'un tube de transport du gaz naturel en polyéthylène de haute densité (HDPE). L'approche proposée utilise un filament continu et uniforme, automatiquement usiné du tube à faible vitesse de coupe et à profondeur de passe optimale afin de réduire le flux de chaleur ainsi que les modifications structurales. Des courbes typiques $(\sigma-\varepsilon)$, dans chaque couche, sont obtenues sur une machine d'essai conçue pour les polymères et sont statistiquement analysées. Elles montrent que le comportement mécanique du tube en HDPE est pratiquement divisé en 3 zones distinctes dont la seconde reste la plus étendue. Le niveau de contrainte moyen exprimant l'étirage à froid pour une couche donnée est presque constant à travers l'épaisseur du tube. Les contraintes et le module d'élasticité donnent de très bonnes corrélations avec l'épaisseur indiquant une augmentation de la surface externe vers les couches internes. Ceci est expliqué par l'évolution de cristallinité durant le procédé de production faisant appel à un refroidissement échelonné dans le temps et qui génère des contraintes résiduelles. Les corrélations statistiques à l'écoulement plastique, au début de l'étirage à froid et à la rupture indiquent des relations linéaires acceptables pour une probabilité d'erreur $p \leq 0,05$. D'autre part, une corrélation linéaire croissante caractérise la relation entre la contrainte limite et le module d'élasticité. Ce résultat est confirmé dans la littérature pour des éprouvettes standards obtenues par moulage en compression.
\end{abstract}

Mots clés : Tube en polyéthylène / module d'élasticité / cristallinité / étirage / orientation

Abstract - Experimental study of mechanical and morphological properties in HDPE-80 gas pipe. The objective of the experimental study is to establish mechanical and morphological properties distribution throughout a high-density polyethylene gas pipe wall. A continuous and uniform filament is automatically machined from the pipe at a very low cutting speed and an optimal depth of cut to minimize heating and structure disturbances. Typical engineering $(\sigma-\varepsilon)$ curves, in every layer, are obtained using a testing machine especially designed for polymers and are statistically analyzed. Stress-strain behavior of HDPE pipe material is basically divided into 3 distinctive zones of which the second remains the important. The average stress level illustrating cold drawing for a given layer was almost constant throughout the pipe wall. Measured stresses and modulus correlated very well with pipe thickness indicating an increase from outer towards inner layers. This is explained from cristallinity evolution as the pipe production process is subjected to convective water-cooling, which generates residual stresses. Computed statistical stress-strain correlations at yielding, onset of cold drawing and fracture points revealed acceptable linear relations for an error level $p \leq 0.05$. On the other hand, an increasing linear correlation is found to characterize the relation of yield stress to elastic modulus. This result is confirmed from literature for standard specimens prepared by compression molding.

Key words: Polyethylene pipe / elastic modulus / cristallinity / drawing / orientation

\footnotetext{
a Auteur correspondant :

chaoui@univ-annaba.org; chaoui_k@yahoo.fr
} 


\section{Introduction}

De nos jours, les résines de polyéthylène de haute densité (HDPE) sont généralement transformées en tubes et assemblages à grande échelle pour construire des réseaux de transport et de distribution de gaz naturel. Des statistiques récentes montrent que plus de $90 \%$ de systèmes de gaz nouvellement installés dans le monde entier sont exclusivement faits en polyéthylène en raison de son coût relativement faible, sa facilité d'installation et de maintenance et de sa durabilité à long terme vis-à-vis de la dégradation due aux effets de l'environnement, par rapport aux systèmes métalliques [1-4]. En dépit de l'acceptation du polyéthylène $(\mathrm{PE})$ comme alternative économique, la sûreté de fonctionnement est restée une question fondamentale particulièrement dans les cas de rupture semi-fragile à long-terme et de fissuration accélérée par corrosion sous contrainte dans des systèmes de distribution d'eau et de gaz. Il est possible de contrôler dans une certaine mesure les propriétés physiques des polymères semi-cristallins par le management de la morphologie pendant les transformations [5-9]. Une amélioration sensible dans le module de Young et la résistance à la traction a été obtenue par une orientation due à un cisaillement contrôlé dans le cas du moulage par injection et le cas de l'injection à haute pression en raison de l'aspect des structures finales fortement orientées [10]. L'essai mécanique quasi-statique de HDPE traité par SCORIM a montré une amélioration de $59 \%$ dans le module en flexion par rapport à la performance mécanique des éprouvettes de moulage conventionnel par injection [11].

Dans cet article, nous avons essayé de concevoir un procédé expérimental simple afin d'établir la distribution de propriétés mécaniques à travers la paroi d'un tube en HDPE. L'idée consiste à préparer des éprouvettes en fonction de la position de la couche appartenant à une enveloppe de tube, montrer qu'elles sont conformes pour mesurer des propriétés mécaniques de façon reproductible et enfin, construire des corrélations relatives à l'hétérogénéité des tubes en raison des effets thermomécaniques. Comme la production et l'utilisation des tubes en HDPE impliquent la génération de la chaleur lors du formage et du soudage, les variances morphologiques et les gradients de contraintes existeront sous diverses façons et influenceront différemment les résines de tube durant la vie de service. En conséquence, l'identification et la compréhension de l'évolution des propriétés mécaniques dans le tube lui-même et la corrélation des résultats avec la morphologie et les contraintes internes sont désignées comme objectifs de cette étude.

\section{Bases théoriques}

Dans le cas des tubes extrudés, il est admis que les contraintes résiduelles et les variations de la morphologie découlent principalement du processus de fabrication qui ne permet pas la dissipation thermique progressive [11-13]. La nécessité d'avoir des dimensions géométriques homogènes en termes de diamètre et d'épaisseur, qui sont représentées par le facteur SDR, impose un refroidissement rapide. En conséquence, des contraintes de compression dans le processus d'extrusion sont générées sur les couches externes du tube tandis que les couches internes développent des contraintes positives. La résistance à la propagation de fissure est amplement influencée par l'état et la magnitude de ces contraintes résiduelles. En outre, il a été montré que la propagation de fissures est plus lente dans les couches externes soumises à des contraintes résiduelles de compression [14].

Une autre question importante concernant la propagation lente de fissures ( $\mathrm{SCG}$ ) pour les tubes en polyéthylène est montrée par les courbes contrainte-temps de rupture composées de deux taux différents correspondant aux mécanismes de rupture ductile et fragile; courbe «type genou ». Le comportement mécanique à long-terme associé à la rupture semi-fragile est le plus craint car il a lieu sans aucun signe présageant. En même temps, la résistance à long-terme est sensiblement diminuée de sorte qu'une prédiction basée sur une linéarité du comportement à court terme ne soit plus applicable. En conséquence, des conceptions d'essais appropriés ont été développées pour étudier la propagation de fissure sur des matériaux de tubes réels sous les modes de fluage et de fatigue dans le but de retrouver par ailleurs des corrélations entre les divers mécanismes de rupture [4, 15-20]. En utilisant une propagation de fissuration sous fatigue à $K_{\text {Imax }}=1,3 \mathrm{MPa}$ et $R=0,1$, des différences de base dans la configuration de la zone d'endommagement entre les tubes à résines HDPE et MDPE ont été identifiées par des examens au MEB [21]. Ces observations critiques sont précieuses car la zone d'endommagement représente les endroits où la plupart des événements hiérarchiques se produisent et automatiquement participeront au contrôle du taux de propagation de la fissure. Plus tard, ce contrôle aura défini le niveau de résistance ultime à la rupture à long-terme. Sachant que la majeure partie de la durée de vie du tube est contrôlée par la période d'initiation et puis, en identifiant la zone d'endommagement au fond de l'entaille, il a été observé des craquelures simples et d'autres ayant la forme d'epsilon respectivement dans les tubes en HDPE et en MDPE. Il a été conclu que les morphologies correspondantes entretiennent des différences drastiques dans l'organisation du matériau fortement étiré. Tandis que la craquelure simple de HDPE est accommodée en texture fibreuse uniaxialement alignée, la craquelure multiple de MDPE est seulement formée par des micro-vides et des plans biaxialement étirés [16,19,21].

À ce stade, il est nécessaire de corréler les propriétés mécaniques usuelles mesurées macroscopiquement avec celles de la structure associée à la résine. En d'autres termes, il faut pouvoir expliquer les propriétés physiques et mécaniques du matériau par rapport à l'architecture moléculaire et au comportement viscoélastique [8,11]. La rupture fragile des polymères semi-cristallins a été supposée provenir de la séparation des chaînes en des fibrilles et récemment, d'autres études ont également conclu sur la rupture des chaînes due à la contrainte appliquée pendant la propagation de fissure faisant intervenir la fibrillation 
dans la zone d'endommagement $[14,15,20,22]$. Une certaine complexité se situe au niveau de l'hétérogénéité intramoléculaire de la distribution des co-unités qui devrait être en principe aussi efficace que l'hétérogénéité intermoléculaire pour produire des liens moléculaires et des repliements aléatoires de chaînes aux dépens du pliage régulier de chaînes $[5,8]$. Il est admis que les zones cristallisées dans les polymères semi-cristallins influencent fortement les propriétés obtenues à faibles taux de déformation comme le module d'élasticité, la contrainte d'écoulement, la fissuration lente (SCG) et la fissuration sous contrainte dans un environnement agressif (ESC). À l'opposé, les propriétés associées à des taux de déformation élevés telles que l'impact, la déchirure et la propagation rapide de fissure (RCP) sont principalement contrôlées par les régions amorphes de la structure [23,24]. Dans plusieurs cas, des techniques complémentaires ont été adoptées pour évaluer l'information moléculaire à partir des mesures de propriétés physiques. Par exemple, la ténacité à la rupture fragile, les criques, les mesures de SCG ont été utilisées pour estimer la concentration de liens moléculaires et pour en déduire d'importantes informations structurales [25]. Dans des cas plus simples, les concentrations en liens moléculaires ont été évaluées à partir du module du matériau mécaniquement orienté. Les expériences en état d'écoulement supposent que ces molécules-liens et les chaînes empêtrées se comportent comme un réseau caoutchouteux, tandis que dans les régions étirées et durcies; les molécules sont de plus en plus tendues à partir de lamelles fragmentées pour devenir une partie d'un domaine amorphe orienté [8]. Les propriétés mécaniques du tube en plastique sont également affectées par ces variations morphologiques de la matière. Pour illustration, la résistance mécanique des polymères dépend de la rigidité des chaînes et de la concentration des segments moléculaires intra et intercristallins qui fournissent la résistance globale du polymère aux charges externes. Cette résistance est fortement affectée par les températures de traitement et de service pendant le transport du gaz naturel pressurisé. En conséquence, les températures de transition vitreuse et de fusion deviennent des paramètres importants qui conditionnent les opportunités d'utilisation et de travail des tubes en HDPE. Ce matériau doit être renforcé dans deux directions : circonférenciellement pour améliorer son comportement sous l'effet de la pression et longitudinalement afin de préserver une tenue mécanique et une ténacité suffisantes vis-à-vis des chargements des réseaux. La plupart des équations font intervenir les dimensions géométriques telles que le diamètre extérieur $(O D)$ et l'épaisseur $(h)$ en liaison avec les propriétés mécaniques des tubes plastiques. Par exemple, comme indiqué par la norme française NF-EN 921 pour les essais du tube plastique pressurisé, la contrainte circonférencielle est calculée à partir de l'équation de Lamé :

$$
\sigma_{\text {hoop }}=P \frac{O D-h}{2 h}
$$

De l'autre côté, pour calculer la contrainte résiduelle maximale dans les tubes plastiques, la relation suivante est une approximation reliant le module de fluage au temps $t$ et à l'épaisseur du tube [26] :

$$
\sigma_{\max }=\frac{ \pm E(t) h}{1-\nu^{2}} \cdot \frac{D_{2}(t)-D_{1}}{D_{2}(t) D_{1}}
$$

où $D_{1}$ et $D_{2}$ sont les diamètres du tube avant et après le sectionnement de l'anneau. Alternativement, la contrainte $\sigma$ agissant sur la paroi du tube devrait obéir à une équation de la forme suivante afin d'assurer les conditions de sûreté en fonctionnement [27] :

$$
\sigma^{2} \geq \frac{2\left(K_{\mathrm{c}}\right)^{2}\left(1-\nu^{2}\right)}{\pi D}
$$

où $D$ est le diamètre moyen et $K_{\mathrm{c}}$ est la ténacité du matériau. L'autre étape est le dimensionnement d'essais concernant les tubes en HDPE dans le but d'investiguer les corrélations des propriétés mécaniques avec les paramètres de la morphologie tels que la cristalinité $X$, le poids moléculaire $M_{0}$ et le nombre moyen de segments dans un lien moléculaire représenté par $N$. Krigbaum et al. ont réussi à dériver et à appliquer, dans le cas d'un polymère sphérulitique en déformation, une expression du module d'élasticité initial $E$ sous la forme [28] :

$$
E=\frac{\rho R T}{M_{0}}\left[\frac{1}{5} \frac{1}{N(1-X)^{3}} \frac{\beta^{2} \sinh \beta}{\sinh \beta-\beta^{2}}+\frac{4}{5} \frac{\beta}{N^{1 / 2}(1-X)}\right]
$$

et

$$
\frac{\sinh \beta}{\beta}=\exp \left[\frac{\Delta H_{f}}{R}\left(\frac{1}{T}-\frac{1}{T_{\mathrm{m}}^{0}}\right)\right]
$$

Tous les symboles ont leur signification habituelle et à partir de la relation (4), l'influence directe de la morphologie avec $X, N$ et $\rho$ sur les propriétés mécaniques peut être appréciée. Une autre approche a été aussi adoptée pour estimer la valeur du module suivant la direction d'une chaîne de polymère qui se cristallise essentiellement sous la forme d'un zigzag plan où sont impliquées seulement les déformations dues au recourbement et à l'étirement des liaisons. Pour un polymère composé de $n$ tiges, chacune de longueur $l$ et l'ensemble joint par des ressorts de torsion, si $\theta$ est l'angle de liaison et $A$ est la section normale supportée par chaque chaîne alors, le module d'élasticité est dérivé comme suit [29]:

$$
E=\frac{l \sin (\theta / 2)}{A}\left[\frac{\sin (\theta / 2)}{k_{1}}+\frac{l^{2} \cos ^{2}(\theta / 2)}{4 k_{\theta}}\right]^{-1}
$$

où $k_{1}$ et $k_{\theta}$ sont des constantes corresponds respectivement à l'étirement de la liaison et à la force de déformation angulaire. Cependant, des approches plus simples sont recherchées pour une analyse immédiate dans le cas des tubes plastiques.

\section{Approche expérimentale}

\subsection{Matériau}

Le matériau utilisé dans cette étude est un tube en HDPE extrudé à partir d'une résine PE 80 par Europlast 
conformément aux conditions générales de qualité et d'essai DIN 8075. Selon ISO 9080, la résistance minimale exigée (MRS) pour cette résine est dans la fourchette 6,3 à $8 \mathrm{MPa}$ sur la base d'une période assurée de 50 ans en service. Le MFI et la densité du matériau pigmenté sont 0,9 et 0,954 respectivement. Sonelgaz, l'Entreprise Nationale Algérienne de l'Électricité et de Gaz a cordialement fourni des spécimens de tube, avec un $D_{\text {moyen }}$ externe de $125 \mathrm{~mm}$ et d'une épaisseur moyenne de 11,4 mm (SDR 11). Ce tube est utilisé dans des environnements relativement modérés à une pression moyenne de 4,0 bars effectifs en service et peut supporter des pressions d'essai 1,5 fois plus importantes.

\subsection{Préparation des éprouvettes}

Afin de mesurer les propriétés mécaniques dans chaque couche du cylindre, il est demandé de préparer des éprouvettes avec les critères suivants : (a) directement extraites à partir du tube pour conserver l'histoire thermomécanique intrinsèque, (b) devraient obéir à une méthodologie de préparation reproductible et (c) les perturbations structurales de la morphologie doivent être minimisées par la réduction des contraintes de contact durant l'opération automatique d'usinage. Plusieurs conditions de coupe ont été essayées en utilisant un programme d'usinage pour obtenir une section de filament la plus régulière possible à travers la paroi tube. Un mandrin en bois ajusté au diamètre intérieur est fabriqué pour maintenir le tube et les contraintes d'ancrage sont uniformément distribuées par l'utilisation de 3 larges coussinets métalliques entre la surface externe du tube et les mâchoires. Pour éviter tout déplacement radial du montage rotatif, le mandrin a été également soutenu par une poupée mobile. La coupe de filament a été réalisée en continu dans la direction radiale à l'aide d'un outil droit en acier au carbone, de $2 \mathrm{~mm}$ d'épaisseur et à 45 t. $\mathrm{min}^{-1}$. Pour garder les efforts de coupe à un niveau bas, la profondeur de passe a été limitée à $0,5 \mathrm{~mm}$ et puisque l'usinage est orthogonal, l'avance de l'outil est prise nulle. L'examen microscopique a permis de vérifier l'uniformité globale du filament et de la section rectangulaire. Comme l'opération d'enlèvement de matière peut introduire des déformations, toutes les éprouvettes ont été préparées au cours d'une même opération et exactement dans les mêmes conditions. Puisque l'objectif visé est la recherche d'écarts éventuels, chaque filament a permis la préparation d'au moins 50 éprouvettes de $150 \mathrm{~mm}$ de longueur chacune (Fig. 1). L'éprouvette est identifiée géométriquement et spatialement dans la paroi du tube selon le cylindre initial pris comme référence et en utilisant une approche volumétrique [30].

\subsection{Procédure expérimentale}

Les éprouvettes ont été soumises à des essais de traction monotones avec une machine d'essai universelle Zwick Materialprüfung, Type Zwicki 1120 particulièrement conçue pour la caractérisation des polymères
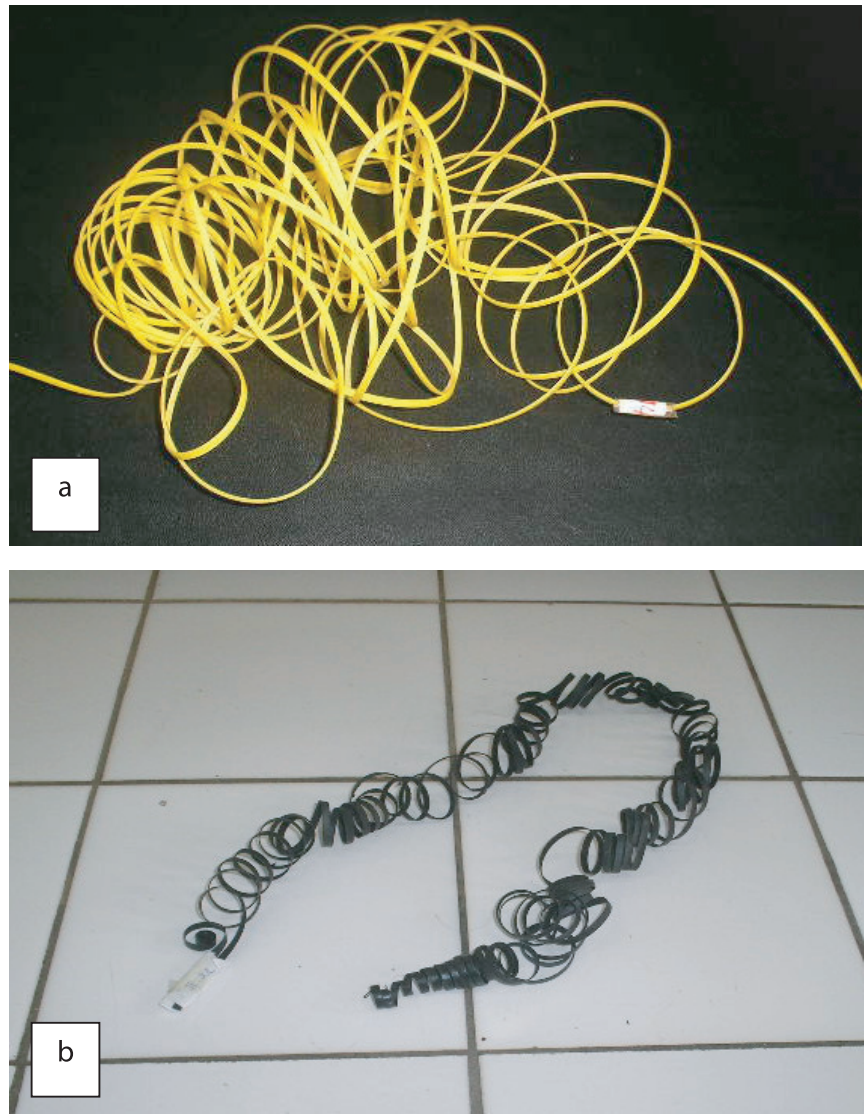

Fig. 1. Filaments en polyéthylène de haute densité, résine 80 usinés à partir d'un tube de gaz de $125 \mathrm{~mm}$ de diamètre, a) direction longitudinale (pigment jaune) et b) direction radiale (pigment noir).

avec une cellule de charge de $2 \mathrm{kN}$. Une vitesse d'essai de 1,66 mm.s ${ }^{-1}$ a été utilisée. Le déroulement des essais a été contrôlé par le logiciel TestXpert ${ }^{\circledR}$ version 6.0 qui a permis de les effectuer exactement de la même manière en se basant sur les recommandations générales de ASTM D-638. Afin d'atteindre la rupture finale du filament, la longueur calibrée a été réduite de $64 \mathrm{~mm}$ à $40 \mathrm{~mm}$ pour adapter la géométrie de l'éprouvette avec la course maximale de la machine et une telle longueur a été également utilisée en littérature [31]. L'acquisition des données sur ordinateur a été assurée en temps réel par une interface RS232. L'analyse statistique instantanée a été réalisée sur demande pendant chaque acquisition de données suivant un programme d'essai précédemment déclaré. Cette étape a assuré le calcul des moyennes et des écarts-type pour chaque ensemble d'éprouvettes appartenant à une même couche et constituant la courbe moyenne contrainte-déformation pour une épaisseur adimensionnelle donnée du tube comme représenté dans la figure 2. 109 éprouvettes ont été testées à la température ambiante pour deux lots identiquement préparés (lot 1 et lot 2). 


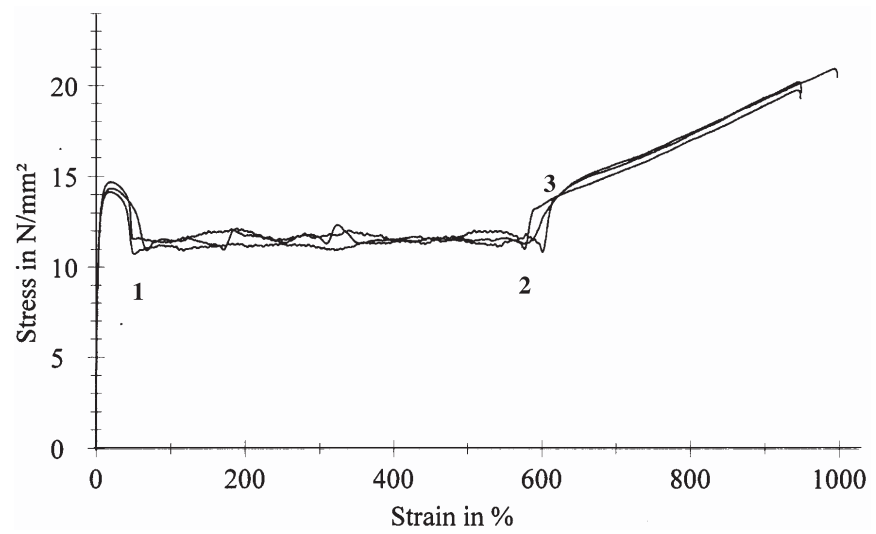

Fig. 2. Courbes $\sigma-\varepsilon$ de 3 éprouvettes HDPE appartenant à une même couche obtenues par le logiciel TestXpert. (1) Début d'étirage à froid, (2) fin d'étirage et (3) début du durcissement plastique.

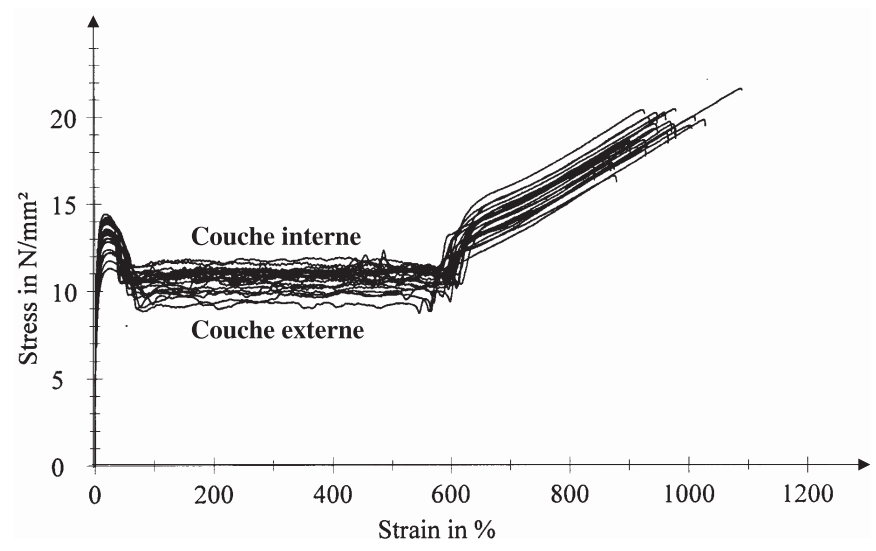

Fig. 3. Comparaison des courbes $\sigma-\varepsilon$ obtenues pour le lot 1 couvrant l'ensemble de l'épaisseur du tube en HDPE (24 éprouvettes).

\section{Résultats}

La figure 3 illustre un ensemble de 24 courbes moyennes $(\sigma-\varepsilon)$ typiques délivrées par le rapport du logiciel TestXpert ${ }^{\circledR}$. Trois zones distinctives caractérisent le comportement : (a) une région élastique linéaire, (b) une région d'étirage à froid montrant plus de $500 \%$ de déformation et (c) une déchirure ultime du matériau associée à la rupture finale. Cette courbe identifie le comportement typique des polymères semi-cristallins, qui sont généralement plus ductiles particulièrement entre $T_{\mathrm{g}}$ et $T_{\mathrm{m}}$ et subissent l'étirage à froid avant la rupture ultime. Les observations attentives indiquent que l'étirage à froid commence juste après le point d'écoulement et avant le point 1 (Fig. 2). Après le point 2, le durcissement plastique a lieu provoquant l'augmentation de la contrainte et les cristallites finissent par se fragmenter en une structure fibreuse fortement anisotrope alignée dans le sens d'étirement [28-31]. Afin d'étayer les variations dans la paroi du tube, la comparaison de courbes $(\sigma-\varepsilon)$ correspondantes à toutes les positions moyennes montre que l'allure générale est très semblable et les 3 zones observées

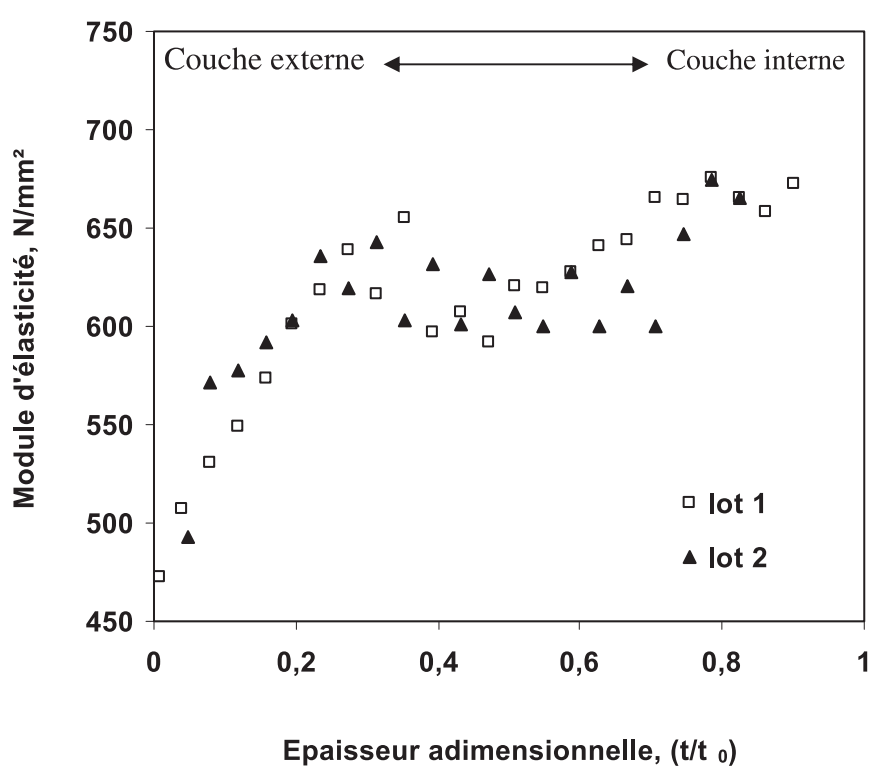

Fig. 4. Évolution du module d'élasticité à travers la paroi d'un tube de gaz en HDPE pour deux lots préparés de manière identique.

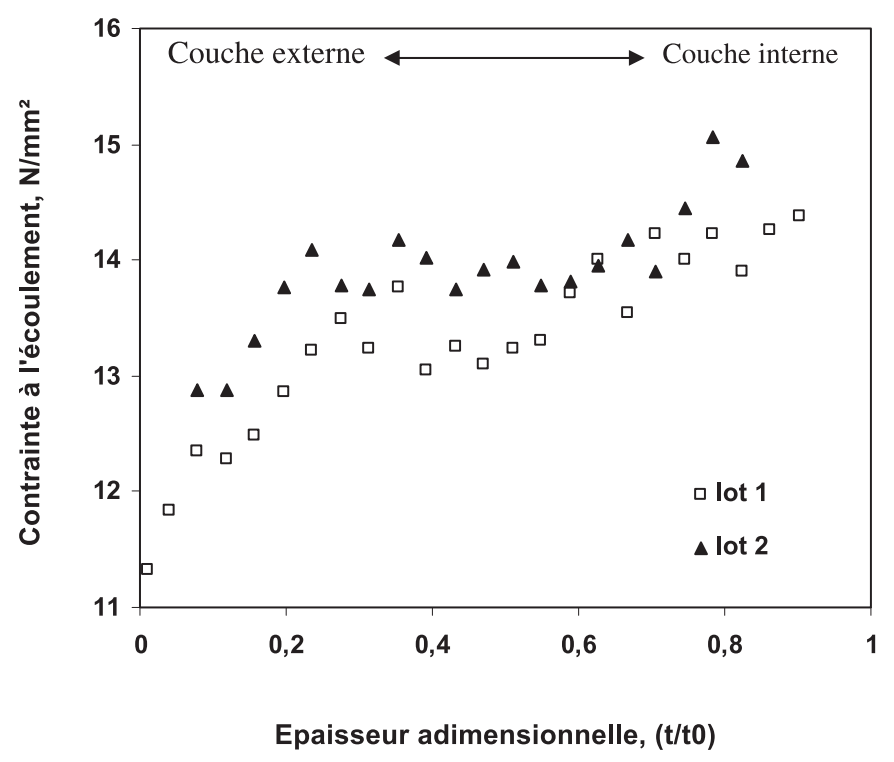

Fig. 5. Évolution du $\sigma_{y}$ à travers la paroi du tube pour deux lots identiques.

sont préservées. En plus, il est remarqué qu'une tendance est établie à mesure que les courbes évoluent de la couche interne vers l'extérieur (Fig. 3). Pour étudier cette variabilité, les propriétés mécaniques courantes sont calculées et enregistrées par le logiciel pour chaque couche de l'enveloppe.

Pour le module d'élasticité et la contrainte d'écoulement (Figs. 4 et 5), il y a une augmentation apparente des 2 propriétés en allant de l'extérieur vers les couches internes du tube. Dans la région s'étendant entre 30 et $70 \%$ d'épaisseur du tube, un plateau est observé indiquant probablement une zone qui n'a pas été complètement affectée par le transfert 


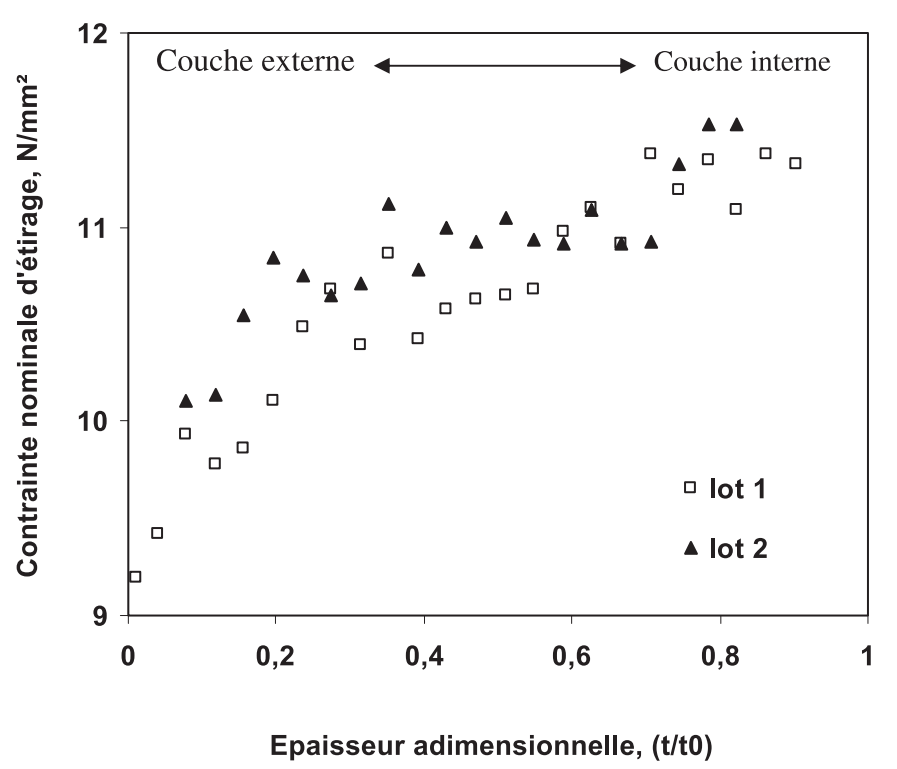

Fig. 6. Évolution de la contrainte nominale d'étirage dans la paroi du tube.

thermique pendant l'extrusion particulièrement pour les tubes obtenus par extrusion et rapidement refroidis à l'eau à partir de la surface externe [30]. De l'autre côté, la surface intérieure a eu suffisamment de temps pour se refroidir par convection libre. La plupart des équations prévoient une augmentation de $E$ et de $\sigma_{y}$ avec la cristalinité mais dans ce cas, il est impératif de souligner que le cas du cylindre est différent car un gradient de température contrôle le système thermodynamique transitoire pendant le refroidissement. Cette déduction est effective pour le schéma lamellaire moyen de Boyd et l'équation semi-empirique de Tsai-Halpin [28, 29, 32, 33]. Il est possible avec les données obtenues d'écrire les équations polynômes suivantes exprimant les variations du module d'élasticité et de la contrainte d'écoulement en fonction de l'épaisseur :

$$
\begin{aligned}
E= & 1349,80\left(\frac{t}{t_{0}}\right)^{3}-2002,70\left(\frac{t}{t_{0}}\right)^{2} \\
& +960,87\left(\frac{t}{t_{0}}\right)+475,75 \\
\sigma_{y}= & 15,55\left(\frac{t}{t_{0}}\right)^{3}-23,80\left(\frac{t}{t_{0}}\right)^{2}+12,49\left(\frac{t}{t_{0}}\right)+11,538
\end{aligned}
$$

La figure 6 montre une observation semblable pour la contrainte nominale d'étirage puisqu'elle croît également dans la même direction. La moyenne et l'écart type calculés sont $10,73 \mathrm{~N} . \mathrm{mm}^{-2}$ et $0,53 \mathrm{~N} \cdot \mathrm{mm}^{-2}$ respectivement pour les deux lots testés. Il a été observé une consistance dans les mesures probablement contrôlée par une hiérarchie structurale elle-même régie par la cristallinité durant le procédé d'écoulement à volume constant. Similairement, la contrainte moyenne d'étirage a été calculée

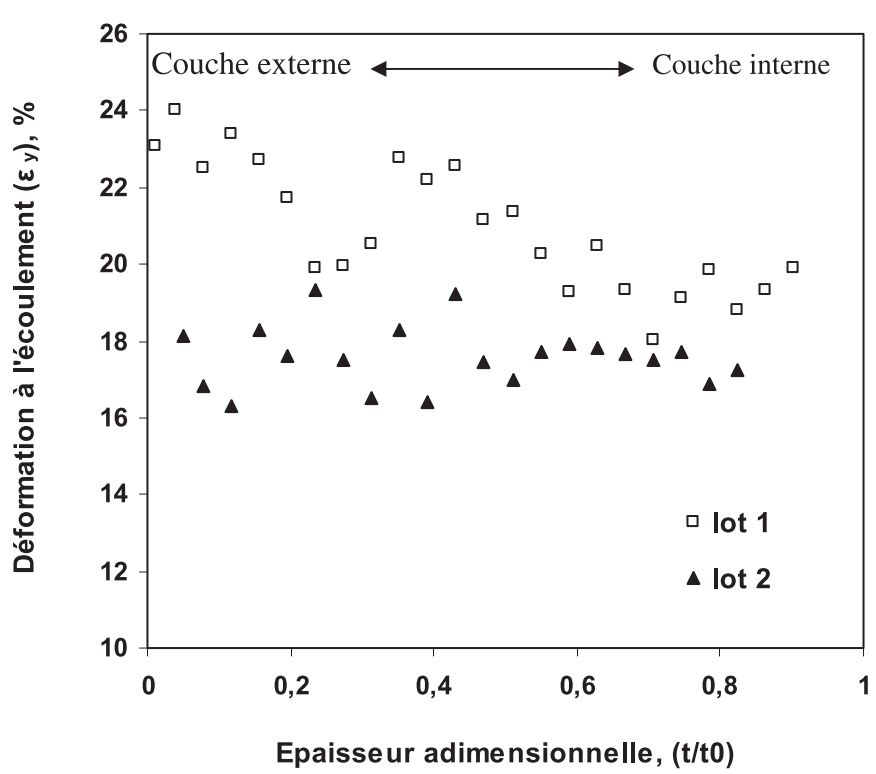

Fig. 7. Évolution de la déformation à l'écoulement en fonction de l'épaisseur adimensionnelle du tube.

en fonction de l'épaisseur et la relation suivante en donne une bonne description :

$$
\overline{\sigma_{\mathrm{CD}}}=8,47\left(\frac{t}{t_{0}}\right)^{3}-13,53\left(\frac{t}{t_{0}}\right)^{2}+7,85\left(\frac{t}{t_{0}}\right)+9,28
$$

Pour les mesures de déformation, les corrélations n'ont pas été évidentes à la limite élastique, au début de l'écoulement et à la rupture. Les dispersions en déformation dans toute l'épaisseur du tube sont importantes mais la reproductibilité des résultats est maintenue pour $\varepsilon_{y}$ et $\varepsilon_{\mathrm{f}}$ dans les 2 lots (Figs. 7 et 8 ). L'étendue du palier d'étirage à froid exprimée en \% est illustrée dans la figure 9. Les basses valeurs mesurées ont lieu sur les couches externes du tube mais au-delà de la limite de $20 \%$ d'épaisseur, une dispersion appréciable existe dans les 2 lots entre 480 et 580 \%. Dans cette deuxième zone, l'étirage à froid se produit par deux mécanismes. Dans le premier cas, il s'initie au milieu de l'éprouvette en un seul endroit et la striction conséquente se propage progressivement vers les mors. Alternativement, dans d'autres cas, le début d'étirage est caractérisé par une multitude de sites soumis au phénomène de la striction se développant indépendamment et puis, fusionnant ensemble. Pendant ce processus, la courbe $(\sigma-\varepsilon)$ en temps réel a montré des fluctuations localisées caractéristiques qui ont décrit l'activité de l'étirage associée (Fig. 2). Pendant cet écoulement, les cristallites deviennent plastiquement déformés et subissent un cisaillement plastique localisé dans des plans de glissement.

Le durcissement plastique est dû pour une faible part à la consolidation de la plasticité cristalline, mais davantage aux effets entropiques de l'orientation moléculaire dans la phase amorphe et puis, dans les cristallites eux-mêmes. Aux grandes déformations plastiques, les chaînes subissent aussi une orientation progressive de sorte que leur distorsion ultérieure devienne de plus 


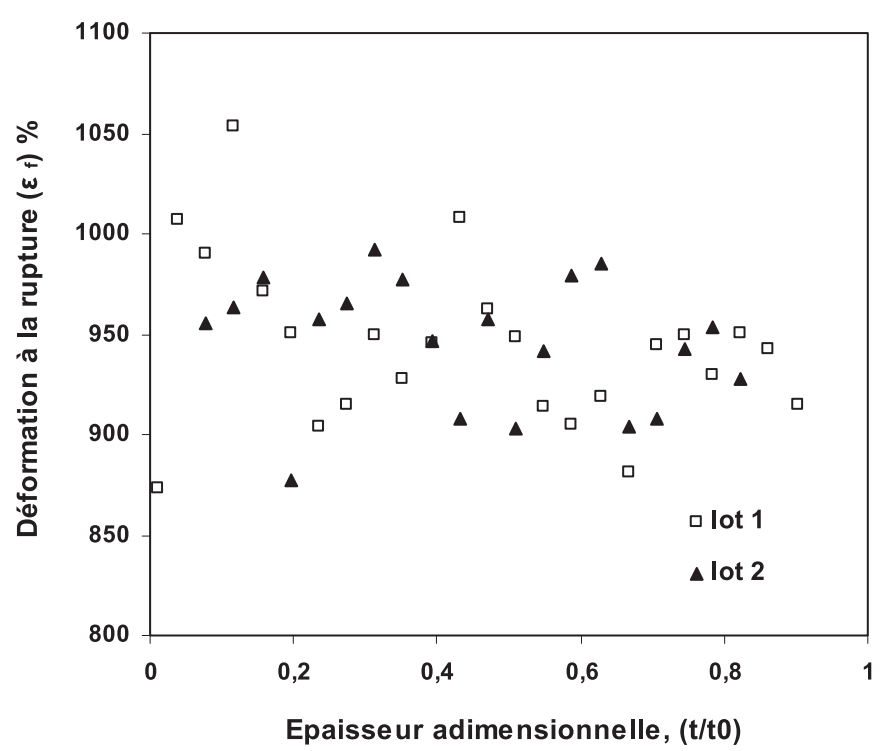

Fig. 8. Évolution de la déformation à la rupture en fonction de l'épaisseur du tube.

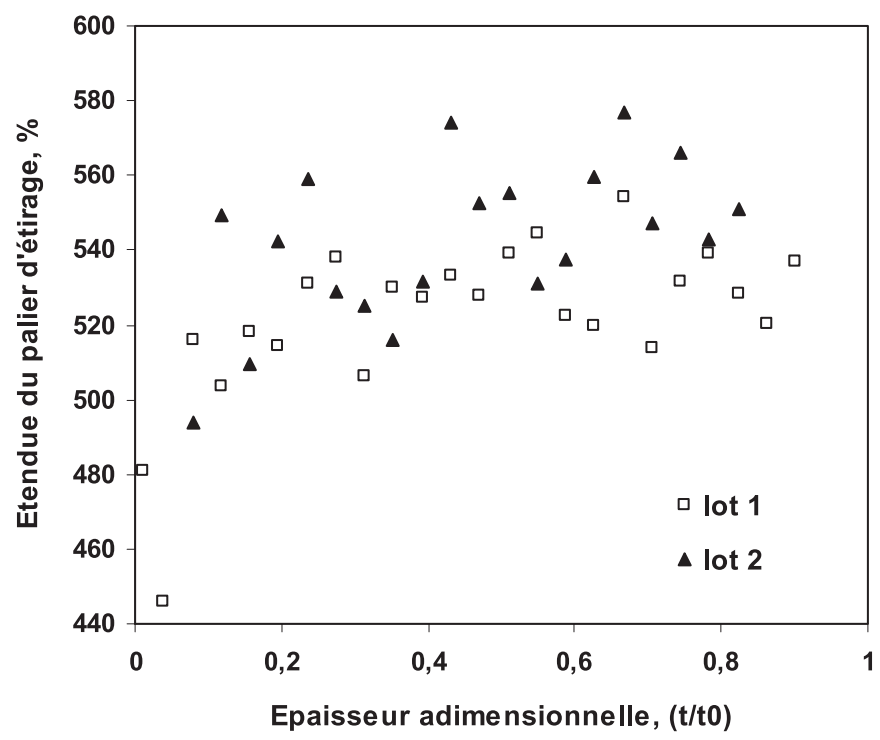

Fig. 9. Étendue du palier d'étirage $\left(\Delta \varepsilon_{C D}\right)$ en fonction de l'épaisseur du tube.

en plus difficile. Ceci nécessite une augmentation de la contrainte d'étirement et les cristallites finissent par se fragmenter en une texture fibreuse fortement anisotrope alignée dans la direction de traction. La rupture finale des fibres se produit par des déformations à grandes échelles et des fibres localement craquelées [28,31].

Une analyse statistique a été effectuée sur les données obtenues afin de valider les tendances et d'examiner si des différences significatives existent entre les lots étudiés. Toutes les données ont été soumises à la distribution normale et la dispersion autour de la moyenne a été analysée. Deux points caractéristiques représentés par $\left(\sigma_{y}\right.$, $\varepsilon_{y}$ ) et le début d'étirage (point 1 dans la Fig. 2) sont considérés. Les valeurs moyennes et les écarts-type ont été calculés à partir des figures 10 et 11. Une zone fermée

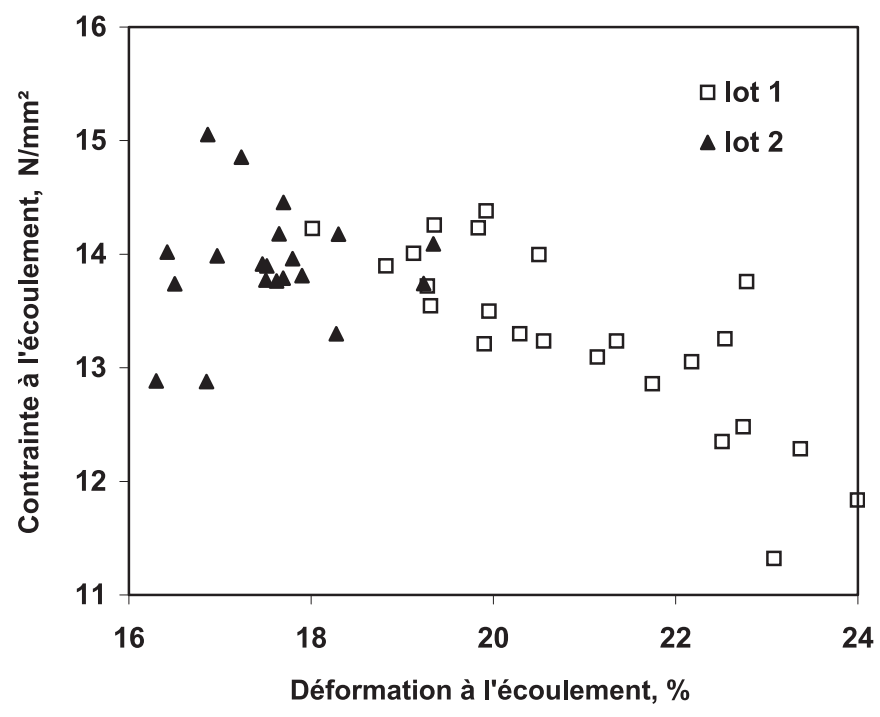

Fig. 10. Relation entre $\sigma$ et $\varepsilon$ à l'écoulement pour les 2 lots.

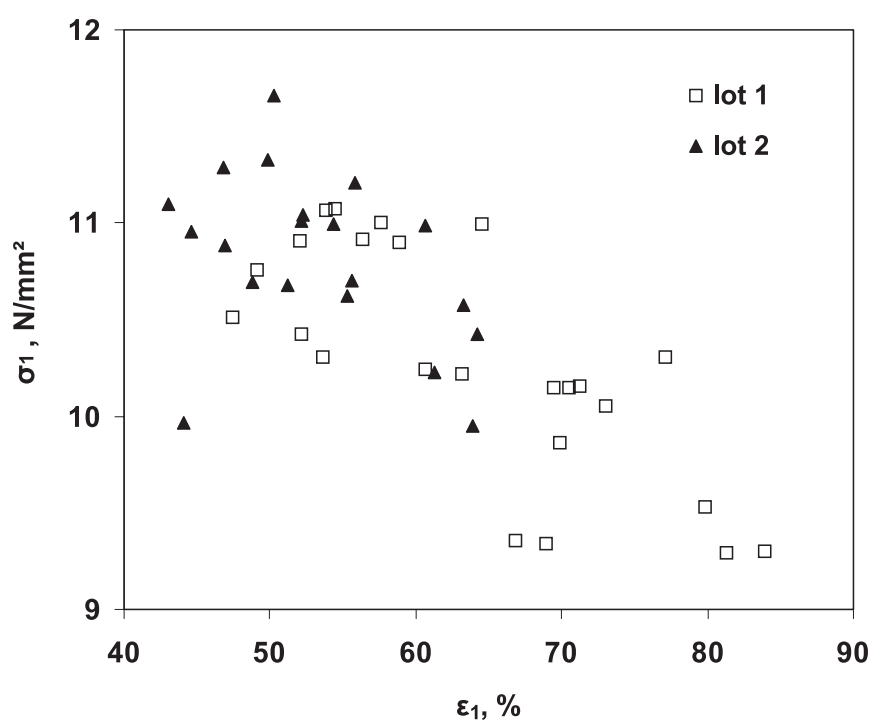

Fig. 11. Relation entre $\sigma$ et $\varepsilon$ au début de l'étirage indiqué par le point 1 dans la figure 2 .

représentant des variations de 2 écarts-type autour de la valeur moyenne est construite pour estimer la constance à travers la paroi en calculant le $\%$ des points circonscrits. Les valeurs obtenues sont $62 \%$ et $57 \%$ respectivement pour les figures 10 et 11, indiquant approximativement que plus de la moitié des données obtenues, accepte bien une distribution normale.

\section{Discussion}

Le comportement $(\sigma-\varepsilon)$ observé est également constaté dans la littérature pour différentes géométries d'éprouvette [28,31,34-37]. L'utilisation des éprouvettes moulées par compression ne peut pas reproduire exactement la structure du tube au moins en termes de contraintes résiduelles. En outre, la préparation des 
Tableau 1. Coefficients de détermination et niveaux de probabilité d'erreur $(p<0,05)$ entre quelques propriétés mécaniques et la cristallinité mesurée par DSC en fonction de l'épaisseur du tube.

\begin{tabular}{|c|c|c|c|c|c|c|c|c|c|}
\hline & Position & $\begin{array}{l}\text { Module } \\
\text { élasticité }\end{array}$ & $\begin{array}{l}\text { Sigma Y. } \\
-0,2 \%\end{array}$ & $\begin{array}{l}\text { Sigma } \\
\text { Max. }\end{array}$ & $\begin{array}{l}\text { Sigma } \\
\text { étirage }\end{array}$ & $\begin{array}{l}\text { Sigma } \\
\text { rupture }\end{array}$ & $\begin{array}{l}\text { Déform. } \\
\text { limite }\end{array}$ & $\begin{array}{l}\text { Déform. } \\
\text { rupture }\end{array}$ & $\begin{array}{l}\text { Déform. } \\
\text { étirage }\end{array}$ \\
\hline $\begin{array}{l}\text { Module } \\
\text { élasticité }\end{array}$ & $\begin{array}{l}0,772^{*} \\
p=0,0001\end{array}$ & - & & & & & & & \\
\hline Sigma Y_0,2\% & $\begin{array}{l}0,725^{*} \\
p=0,0005\end{array}$ & $\begin{array}{l}0,881^{*} \\
p=0,0000\end{array}$ & - & & & & & & \\
\hline Sigma Max. & $\begin{array}{l}0,784^{*} \\
p=0,0004\end{array}$ & $\begin{array}{l}0,878^{*} \\
p=0,0007\end{array}$ & $\begin{array}{l}0,972^{*} \\
p=0,0000\end{array}$ & - & & & & & \\
\hline Sigma étirage & $\begin{array}{l}0,861^{*} \\
p=0,0000\end{array}$ & $\begin{array}{l}0,886^{*} \\
p=0,0005\end{array}$ & $\begin{array}{l}0,929^{*} \\
p=0,0000\end{array}$ & $\begin{array}{l}0,967^{*} \\
p=0,0000\end{array}$ & - & & & & \\
\hline Sigma rupture & $\begin{array}{l}0,343^{*} \\
p=0,0223\end{array}$ & $\begin{array}{l}0,334^{*} \\
p=0,0273\end{array}$ & $\begin{array}{l}0,151 \\
p=0,3271\end{array}$ & $\begin{array}{l}0,199 \\
p=0,1968\end{array}$ & $\begin{array}{l}0,249 \\
p=0,1029\end{array}$ & - & & & \\
\hline Déform. limite & $\begin{array}{l}-0,358^{*} \\
p=0,0177\end{array}$ & $\begin{array}{l}-0,453^{*} \\
p=0,0022\end{array}$ & $\begin{array}{l}-0,718^{*} \\
p=0,0008\end{array}$ & $\begin{array}{l}-0,669^{*} \\
p=0,0009\end{array}$ & $\begin{array}{l}-0,589^{*} \\
p=0,0009\end{array}$ & $\begin{array}{l}0,190 \\
p=0,2170\end{array}$ & - & & \\
\hline $\begin{array}{ll}\text { Déform. rup- } \\
\text { ture }\end{array}$ & $\begin{array}{l}-0,284 \\
p=0,0624\end{array}$ & $\begin{array}{l}-0,224 \\
p=0,1444\end{array}$ & $\begin{array}{l}-0,253 \\
p=0,0982\end{array}$ & $\begin{array}{l}-0,232 \\
p=0,1301\end{array}$ & $\begin{array}{l}-0,281 \\
p=0,0651\end{array}$ & $\begin{array}{l}0,565^{*} \\
p=0,0004\end{array}$ & $\begin{array}{l}0,200 \\
p=0,1930\end{array}$ & - & \\
\hline $\begin{array}{l}\text { Déform. } \\
\text { étirage }\end{array}$ & $\begin{array}{l}0,503^{*} \\
p=0,0003\end{array}$ & $\begin{array}{l}0,538^{*} \\
p=0,0008\end{array}$ & $\begin{array}{l}0,623^{*} \\
p=0,0003\end{array}$ & $\begin{array}{l}0,645^{*} \\
p=0,0005\end{array}$ & $\begin{array}{l}0,632^{*} \\
p=0,0002\end{array}$ & $\begin{array}{l}-0,206 \\
p=0,1796\end{array}$ & $\begin{array}{l}-0,511^{*} \\
p=0,0001\end{array}$ & $\begin{array}{l}-0,349^{*} \\
p=0,0208\end{array}$ & - \\
\hline Cristallinité & $\begin{array}{l}0,923^{*} \\
p=0,0000\end{array}$ & $\begin{array}{l}0,633^{*} \\
p=0,0003\end{array}$ & $\begin{array}{l}0,596^{*} \\
p=0,0005\end{array}$ & $\begin{array}{l}0,643^{*} \\
p=0,0003\end{array}$ & $\begin{array}{l}0,710^{*} \\
p=0,0009\end{array}$ & $\begin{array}{l}0,382^{*} \\
p=0,010\end{array}$ & $\begin{array}{l}-0,270 \\
p=0,0760\end{array}$ & $\begin{array}{l}-0,250 \\
p=0,1020\end{array}$ & $\begin{array}{l}0,306^{*} \\
p=0,0436\end{array}$ \\
\hline
\end{tabular}

(*) indique un niveau d'erreur statistique acceptable.

éprouvettes du tube suivant la norme ASTM implique des opérations prolongées de coupe et d'usinage à travers la paroi, ce qui va perturber profondément la morphologie initiale. Pour un objectif semblable, des courbes $(\sigma-$ $\varepsilon)$ ont été obtenues avec des éprouvettes standardisées soudées par électro-fusion et bout à bout à partir d'un tube en HDPE pour établir les différences sous la forme de $E, \sigma_{y}$ et $\varepsilon_{\mathrm{f}}[35]$. En commun avec cette étude, il est observé que la contrainte de traction ultime a montré une tendance semblable au module d'élasticité (Figs. 4 et 5). Puisque des tubes plus épais sont recherchés pour transporter des débits encore plus élevés à des pressions plus importantes, l'influence de la méthode d'assemblage du tube plastique est une autre variable critique qui doit être étudiée indépendamment et l'approche décrite ici serait appropriée. Pour les tubes HDPE formés par écoulement plastique, les propriétés mécaniques ont été étudiées en fonction du pourcentage de réduction de diamètre sur des éprouvettes structurellement altérées par écrasement. En se référant aux normes ASTM D-2105 et D-1599, il est constaté qu'il y a moins d'écoulement et d'étirage à froid avec l'augmentation du \% réduction du diamètre du tube, tandis que la ténacité évolue positivement selon les directions axiale et circonférencielle [36]. Aussi, des mesures de cristallinité ont été effectuées sur des tubes en polyéthylène de moyenne et haute densités et il a été trouvé qu'un écart allant jusqu'à $8 \%$ existe entre les couches externes et internes. Dans une autre étude, il a été conclu que la durée de vie prédite par les essais à charges constantes (CTL) augmente linéairement avec la cristallinité moyenne [38]. Ces conclusions confortent les résultats de cette approche comme montré dans les figures 3 à 6 . Les corrélations impliquant les déformations $\left(\varepsilon_{y}, \varepsilon_{\mathrm{f}}\right.$ et palier d'étirage à froid) n'ont pas montré des tendances linéaires comme indiqué dans le tableau 1, de l'analyse statistique effectuée sur l'ensemble des données en utilisant le logiciel Statistica ${ }^{\circledR}$ (version 5.1). La qualité de l'ajustement a été examinée avec le test de Fisher-Snedecor pour un niveau de probabilité d'erreur présélectionné de $p \leq 0,05$. Les corrélations acceptées sont indiquées avec une étoile dans le tableau 1. $\mathrm{Au}$ niveau de la morphologie, les mesures de cristallinité indiquent aussi cette évolution spécialement avec les différentes contraintes mesurées, le module d'élasticité et la position à travers la paroi du tube. Par contre, le niveau de correlation est très faible avec les déformations mesurées (Tab. 1).

Afin de confirmer l'utilité d'une telle approche et en se basant sur l'analyse statistique, $E$ et $\sigma_{y}$ montrent une forte corrélation avec l'épaisseur de la paroi du tube comme montré dans la figure 12 . Il est conclu qu'une relation linéaire existe entre ces deux propriétés mécaniques en utilisant cette nouvelle approche en incluant aussi des données de littérature [39-41]. La corrélation régissant cette relation pour le PE 80 est :

$$
\sigma_{y}=0,0152 E+4,2367
$$

Pour les conditions d'essai adoptées ici, les deux propriétés $\sigma_{y}$ et $E$ normalement reflètent la cristallinité $[28,29,39,41]$. Il est connu que $\sigma_{y}$ augmente avec la pression hydrostatique et par conséquent, il y a simultanément une augmentation des modules de cisaillement et d'Young. Comme $\sigma_{y}$ augmente avec la déformation plastique qui développe l'orientation, $E$ également augmente et une telle dépendance est montrée dans quelques travaux [42-44]. En plus des effets structuraux, la variabilité des propriétés mécaniques dans un tube doit être intégrée comme une nouvelle évidence pour évaluer la 


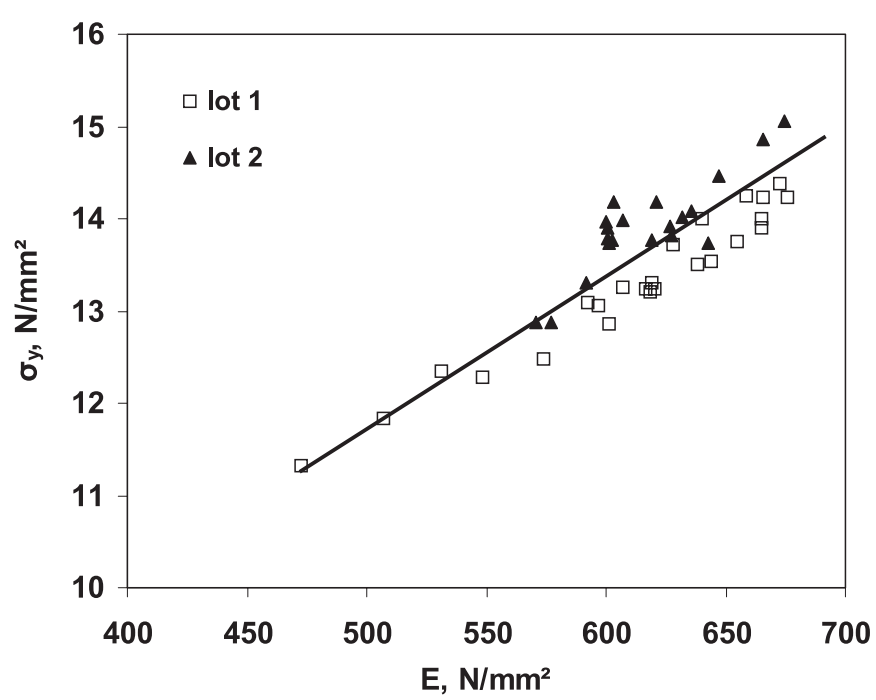

Fig. 12. Relation entre la contrainte limite et le module d'élasticité pour les 2 lots de filaments représentant les différentes couches du tube.

résistance à long-terme et doit être étroitement corrélée avec les contraintes internes, qui peuvent jouer un autre rôle comme celui des contraintes résiduelles compressives contribuant au renforcement de la structure.

\section{Conclusions}

Cette étude a permis d'étudier la distribution des propriétés mécaniques à travers la paroi du tube de gaz en HDPE. Une approche expérimentale est mise en œuvre pour déterminer les différences locales. Bien que, les éprouvettes soient préparées à partir d'un filament continu, les résultats sont en bon accord avec ceux de la littérature. Il est constaté que les propriétés représentant des contraintes augmentent de l'extérieur vers des couches intérieures. Ceci est expliqué par l'évolution de cristalinité puisque le processus de fabrication implique un refroidissement différentiel et aussi une génération de contraintes résiduelles. En termes de déformations, les tendances ne sont pas tout à fait évidentes. Les corrélations contraintedéformation obtenues dans la paroi du tube aux points d'écoulement et au début de l'étirage indiquent une relation linéaire décroissante. D'autre part, la contrainte d'écoulement et le module d'élasticité sont reliés par une forte corrélation linéaire croissante. La variation des propriétés mécaniques à travers la paroi reflète la complexité de la hiérarchie structurelle dans le HDPE et contribue à la compréhension du comportement à long-terme du tube en fonction de la rupture fragile tant redoutée.

Remerciements. Les auteurs sont reconnaissants à l'entreprise SONELGAZ pour la fourniture des d'échantillons de tube HDPE et à l'entreprise pétrolière SONATRACH pour le soutien financier partiel sous le contrat D6001-Z/2000. Le programme expérimental a été effectué au Laboratoire de
Recherche en Mécanique des Matériaux et Maintenance Industrielle (LR3MI) autorisé par le décret MESRS 42/2001.

\section{Références}

[1] Gas Research Institute, Pipeline Statistics, Distribution and Transmission, Annual Mileage Totals, (www.gri.org/pub), Chicago, 2002

[2] J.J. Cheron, Pipeline and Gas Industry 84 (2001) 5

[3] H.B.H. Hamouda, M. Somoes-betbeder, F. Grillon, P. Blouet, N. Billon, R. Piques, Polymer 42 (2001) 5425

[4] C. Munier, E. Gaillard-Devaux, A. Tcharkhtchi, J. Verdu, J. Mater. Sci. 37 (2002) 4159

[5] G. Kalay, R.A. Sousa, R.L. Reis, A.M. Cunha, M.J. Bevis, J. Appl. Polym. Sci. 73 (1999) 2473

[6] A. Keller, H. Kolnaar, Mat. Sci. Technol. 18 (1997) 191

[7] S. Doroudiani, C.B. Park, M.T. Kortschot, Polym. Eng. Sci. 38 (1998) 1205

[8] L. Hubert, L. David, R. Séguéla, G. Vigier, C. Degoulet, Y. Germain, Polymer 42 (2001) 8425

[9] A. Aubert, C. G'Sell, A. Dahoun, E. Dargent, J. Grenet, Revue de Métallurgie, Cahiers d'Informations Techniques 12 (1999) 1511

[10] C.I. Ogbonna, G. Kalay, P.S. Allan, M.J. Bevis, J. Appl. Polym. Sci. 58 (1995) 2131

[11] J.F. Mano, R.A. Sousa, R.L. Reis, A.M. Cunha, M.J. Bevis, Polymer 42 (2001) 6187

[12] K. Chaoui, A. Moet, A. Chudnovsky, in Recent Advances in Experimental Mechanics, J.F. Silva Gomez et al. (eds.), Proceedings of the 10th International Conference on Experimental Mechanics (1994) 811

[13] J.G. William, J.M. Hodgkinson, A. Gray, Polym. Eng. Sci. 21 1981, 822

[14] K. Chaoui, A. Moet, A. Chudnovsky, J. Mater. Sci. 22 (1987) 3879

[15] N. Brown, X. Lu, Y.-L. Huang, R. Qian, Macromol. Chem. 41 (1995) 55

[16] K. Chaoui, J. Mater. Sci. Lett. 8 (1989) 326

[17] M. Parsons, E.V. Stepanov, A. Hiltner, E. Baer, J. Mater. Sci. 35 (2000) 1857

[18] K. Kadota, S. Chum, A. Chudnovsky, J. Appl. Polym. Sci. 49 (1993) 863

[19] J. Strebel, K. Chaoui, A. Chudnovsky, A. Moet, International Gas Research Conference, Tokyo, Paper 218B, 1989

[20] C.J.G. Plummer, A. Goldberg, A. Ghanem, Polymer 42 (2001) 9551

[21] M. Parsons, E.V. Stepanov, A. Hiltner, E. Baer, J. Mater. Sci. 36 (2001) 5747

[22] C.J.G. Plummer, P. Scaramuzzino, H.H. Kaush, Polym. Eng. Sci. 40 (2000) 1306

[23] J.T. Yeh, J.H. Chen, H.S. Hong, J. Appl. Polym. Sci. 54 (1994) 2171

[24] J.T. Yeh, J. Runt, J. Polym. Sci., Part B : Polym. Phys. 29 (1991) 371

[25] M. Ishikawa, K. Ushui, Y. Kondo, K. Hatada, S. Gima, Polymer 37 (1996) 5375

[26] L.J. Broutman, A. Bhatnagar, S. Choi, Residual Stress Studies in Polyethylene Gas Pipes and Fittings, Final Report, GRI Contract 5082-271-0790545, 1986

[27] C.H. Popelar, V.H. Kenner, J.P. Wooster, Polym. Eng. Sci. 31 (1991) 1693 
[28] J.M. Schultz, Polymer Materials Science, Prentice-Hall International Series, New Jersey, 1974, Chap. 11

[29] R.J. Young, Introduction to polymers, Chapman and Hall, New York, 1981

[30] N. Kiass, K. Chaoui, Revue Synthèse des Sciences et de la Technologie, Annaba University 11 (2002) 25

[31] A. Marquez-Lucero, C. G'Sell, K.W. Neale, Polymer 30 (1989) 636

[32] R.H. Boyd, J. Polym. Sci., Part B: Polym. Phys. 21 (1983) 493

[33] R.H. Boyd, Polym. Eng. Sci. 19 (1979) 1010

[34] G.W. Ehrenstein, F. Montagne, Matériaux polymères : Structures, Propriétés et Applications, HERMES Science Publications, Paris, 2000, Chap. 6

[35] H. Chen, R.J. Scavuzzo, T.S. Srivatsan, J. Mater. Sci. 16 (1997) 897

[36] S.H. Teoh, E.H. Ong, Polymer 36 (1995) 101
[37] S.J.K. Ritchie, P. Davis, P.S. Leevers, Polymer 39 (1998) 6657

[38] J.E. Young, L.O. Raphaelian, D.T. Raske, Project Review Meeting, GRI Contract number 5082-271-0717, 1986

[39] B. Crist, C.J. Fisher, P.R. Howard, Macromolecules 22 (1989) 1709

[40] R. Boyd, W. Liau, Macromolecules 19 (1986) 2246

[41] N. Brown, in Failure of Plastics, W. Brostow, R.D. Corneliussen (éds.) SPE, Hanser Publishers, New York, 1986, Chap. 6

[42] A. Peacock, L.J. Mandelkern, Polym. Sci. B, Polym. Phys. 28 (1990) 1917

[43] J. G'Sell, A. Hiver, A. Dahoun, A. Souahi, J. Mater. Sci. 27 (1992) 5031

[44] S. Hobeika, Y. Men, G. Strobl, Macromolecules 33 (2000) 1827 